\title{
The Reduction in Circulating Melatonin Level May Contribute to the Pathogenesis of Ovarian Cancer: A Retrospective Study
}

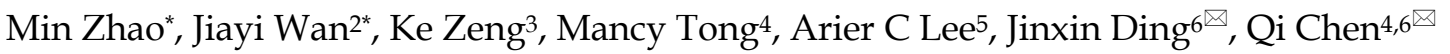 \\ 1. Wuxi Maternity and Children Health Hospital, Nanjing Medical University, China; \\ 2. Department of Pathology, Wuxi No 2 People' Hospital, Nanjing Medical University, China; \\ 3. Department of Orthopaedics, Wuxi No 2 People' Hospital, Nanjing Medical University, China. \\ 4. Department of Obstetrics \& Gynaecology, The University of Auckland, New Zealand; \\ 5. Section of Epidemiology and Biostatistics, School of Population Health, The University of Auckland, New Zealand. \\ 6. The Hospital of Obstetrics \& Gynaecology, Fudan University, China. \\ "Zhao M and Wan JY contributed equally to this work.
}

$\triangle$ Corresponding authors: Dr. Qi Chen, The Hospital of Obstetrics \& Gynaecology, Fudan University, China. Email: q.chen@auckland.ac.nz Phone: 86-13611691734 Or Dr. JX Ding, The Hospital of Obstetrics \& Gynaecology, Fudan University, China. Email: djxdd@sina.com.

( ) Ivyspring International Publisher. Reproduction is permitted for personal, noncommercial use, provided that the article is in whole, unmodified, and properly cited. See http://ivyspring.com/terms for terms and conditions.

Received: 2015.12.01; Accepted: 2016.03.16; Published: 2016.04.27

\begin{abstract}
Ovarian cancer is the third most common gynaecological malignancy. Changes in circadian rhythms such as bright light exposure may affect female reproductive physiology. Night shift work is associated with higher risks of developing gynaecological cancers. In addition, the season of birth is also suggested as an important environmental risk factor for developing gynaecological cancers. Melatonin may play an important role in this association as a marker of circadian rhythms. Serum from 96 women with ovarian cancer and 40 healthy women were collected and the level of melatonin was measured. In addition 277 women with ovarian cancer and 1076 controls were retrospectively collected for season of birth analysis over seven years. The serum levels of melatonin were significantly lower in women with ovarian cancer compared with healthy women $(p<0.05)$. However there was no difference in melatonin levels in perimenopausal and postmenopausal patients. In addition, there is no statistically significant difference in seasonal distribution of birth between ovarian cancer patients and the control group. The melatonin levels in ovarian cancer patients and controls were not associated with the season of birth. Our results demonstrate the lower serum levels of melatonin in ovarian cancer patients which may contribute to the pathogenesis of ovarian cancer. The incidence of ovarian cancer was not associated with the season of birth. The serum levels of melatonin do not appear to be associated with season of birth in ovarian cancer patients.
\end{abstract}

Key words: ovarian cancer, melatonin, season of birth, menopause.

\section{Introduction}

Ovarian cancer is the third most common gynaecological malignancy and the leading cause of death in gynaecological cancers globally and the incidence has been increasing in the last decade [1]. Although the pathogenesis of the ovarian cancer is unclear, studies have suggested that changes in circadian rhythms such as bright light exposure may affect female reproductive physiology [2]. Night shift work is associated with higher risks of developing breast [3-5] and endometrial cancer [6]. Other studies also suggested that the season of birth may be an important environmental risk factor for developing endometrial cancer [7]. However, the mechanism of this association and whether season of birth is also 
associated with developing ovarian cancer remain unknown.

One of the speculations for this association is the reduction in production of melatonin by pineal glands. Melatonin a marker of circadian rhythms is an endogenously-produced lipid soluble hormone whose level changes with circadian rhythm. It has been proposed that melatonin is synthesized in mitochondria at high levels [8] and has multifaceted functions, including direct free radical scavenging. In addition to pineal gland, melatonin is also produced by the ovaries, regulating the function of the ovaries $[9,10]$.

Deficiency of melatonin is associated with the development of many cancers including gynaecological cancers [11, 12]. Supplementation of melatonin has a potential role in cancer prevention through its effects of antioxidant, antiangiogenic activity as well as its ability to modulate the immune system (reviewed in [13]). Recently In vitro studies showed that melatonin has an anti-proliferative effect and inhibits the growth of many cancer cells, including breast cancer, cervical cancer and ovarian cancer and melatonin has effect on controlling cell death [14]. In addition, a recent In vivo study showed that light exposure in the night suppresses the production of melatonin and consequently induces breast cancer insensitive to chemotherapy in rats with breast cancer [15].

The association between melatonin and ovarian cancer has not been fully studied. The reduction of melatonin levels impacts ovary morphology [16]. In vivo studies found that supplementation of melatonin decreases the weight of ovaries in the rat model [17] suggesting melatonin and its metabolites play a direct effect on the reproductive system of women by influencing the function of the ovaries [18]. Taken together these studies may suggest that there is an association between melatonin and the risk of developing ovarian cancer.

Therefore, we undertook this study to investigate the circulating levels of melatonin in women with ovarian cancer. We also investigated whether the season of birth is associated with developing ovarian cancer.

\section{Material and methods}

This study was approved by the Ethics Committee of Wuxi Maternity and Children Hospital, Nanjing Medical University, China. All patient-derived blood samples and tissues were obtained with written informed consent.

\section{Collection of blood samples}

Blood samples from 96 women with ovarian cancer at presentation, and 40 healthy women, were collected by venepuncture into plain Vaccutainer ${ }^{\circledR}$ tubes prior to any treatment between January 2012 and March 2014 at the Wuxi Maternity and Children Health Hospital of Nanjing Medical University of China. The blood was allowed to clot, centrifuged at $2500 \times g$ and the serum was aspirated and stored in aliquots at $-80^{\circ} \mathrm{C}$. Of these 97 women with ovarian cancer, 46 women were post-menopausal. All the blood samples were collected between 8:00-10:00am. The median age for women with ovarian cancer was 48 (range from 18 to 87) years old. Of 96 women with ovarian cancer, there were 59 women with epithelial ovarian cancer, 28 women with sex cord stromal and 9 women with germ cell tumour. The summary of clinical characteristics of 96 women with ovarian cancer for blood collection are summarised in Table 1.

Table 1. Summary of clinical characteristics of women with ovarian cancer.

\begin{tabular}{ll}
\hline & Women with Ovarian cancer (N=96) \\
\hline Median age (years old, range) & $48(18-87)$ \\
With peritoneal metastasis & $59(61.5 \%)$ \\
Without peritoneal metastasis & $37(38.5 \%)$ \\
Perimenopause & $49(50.1 \%)$ \\
Post-menopause & $47(49.9 \%)$ \\
Histology & \\
Epithelial ovarian cancer (number, \%) & $59(61.4 \%)$ \\
Sex cord stromal (number, \%) & $28(29.1 \%)$ \\
Germ cell tumor (number, \%) & $9(9.5 \%)$ \\
\hline
\end{tabular}

\section{Study population for season of birth analysis}

In order to analyse the association between seasons of birth and the incidence of ovarian cancer, data on 277 women with ovarian cancer was retrospectively collected from Wuxi Maternity and Children Health Hospital, Nanjing Medical University of China between January 2008 and December 2014. Data included patients' age, menopause age, date of diagnosis, subtypes of ovarian cancer and peritoneal metastasis. Peritoneal metastasis in epithelial ovarian cancer was identified by macroscopic examination during surgery, and subsequent histological examination of biopsies. Season was defined as winter (December through February), spring (March through May), summer (June through August), and autumn (September through November) based on the climate at Wuxi, China. Wuxi is located close to the East China Sea and it experiences subtropical climate (warm and humid) with four distinct seasons. The duration of sunshine is 9, 16, 6 and 5 hours in spring, summer, autumn and winter respectively. The average temperature is 15,27 , 
18 and $5^{\circ} \mathrm{C}$ in spring, summer, autumn and winter respectively.

In addition, a total of 1,076 healthy age frequency matched women who underwent routine healthy examination were randomly selected from the same hospital during the same period of time. With the ratio of 4 controls per cases, assuming equal probability of birth season among controls and $60 \%$ of cases born in the winter and autumn seasons, 277 cases and 1076 controls will provide at least $80 \%$ power with significance level of 0.05 .

\section{Determination of serum levels of melatonin}

The serum levels of melatonin in women with ovarian cancer and healthy women were measured colorimetrically using a Melatonin ELISA kit (GenWay Biotech Inc, USA; Assay sensitivity is $1.6 \mathrm{pg} / \mathrm{ml}$; intra-assay coefficient of variation is $3.0-11.4 \%$ and inter-assay coefficient of variation is $6.4-19.3 \%$ ) following the manufacturer's instructions.

\section{Statistical analysis}

All data were presented as median and range. The statistical differences in the serum levels of melatonin between women with ovarian cancer and control were analysed by the Mann-Whitney Test using Prism software package. The association of ovarian cancer and season of birth was analysed with a Chi-square test using SAS software version 9.4 (SAS Institute Inc., Cary, NC, USA) with $p<0.05$ being considered as statistically significant.

\section{Results}

\section{The serum levels of melatonin were significantly reduced in women with ovarian cancer}

In order to investigate whether melatonin is involved in the development of ovarian cancer, the serum levels of melatonin in women with ovarian cancer $(n=96)$ were measured. The circulating levels of melatonin were significantly lower in women with ovarian cancer $(41.8 \mathrm{pg} / \mathrm{ml}$ ranging from 1.52 to $223.2 \mathrm{pg} / \mathrm{ml}$ ) compared with healthy women $(82.4$ $\mathrm{pg} / \mathrm{ml}$ ranging from 9.7-241.5pg/ml) (Figure 1, $\mathrm{p}=0.0001$, Mann-Whitney).

We also analysed the difference in the serum levels of melatonin in patients between perimenopause $(n=49)$ and post-menopause $(n=47)$. Interestingly we found there was no significant difference in the levels of melatonin between perimenopausal patients $(44.6 \mathrm{pg} / \mathrm{ml}$, range from 1.54 to $223.1 \mathrm{pg} / \mathrm{ml}$ ) and postmenopausal patients (37.4pg/ml, ranging from 9.3 to $202.2 \mathrm{pg} / \mathrm{ml}$ ) (Figure 2 , $\mathrm{p}=0.274$ ). In addition, we also found that there was no correlation between the serum levels of melatonin and estrogen levels in postmenopausal women with ovarian cancer $(\mathrm{r}=-0.015, \mathrm{p}=0.932)$.

We then analysed the difference in the serum levels of melatonin in patients with $(n=59)$ or without peritoneal metastasis $(n=37)$. We found that there was also no difference in the levels of melatonin between patients with metastasis $(43.6 \mathrm{pg} / \mathrm{ml}$, ranging from 9.3 to $202.2 \mathrm{pg} / \mathrm{ml}$ ) and patients without metastasis $(35.8 \mathrm{pg} / \mathrm{ml}$, ranging from 1.54 to $223.1 \mathrm{pg} / \mathrm{ml}$ ) (Figure $3, \mathrm{p}=0.356)$.

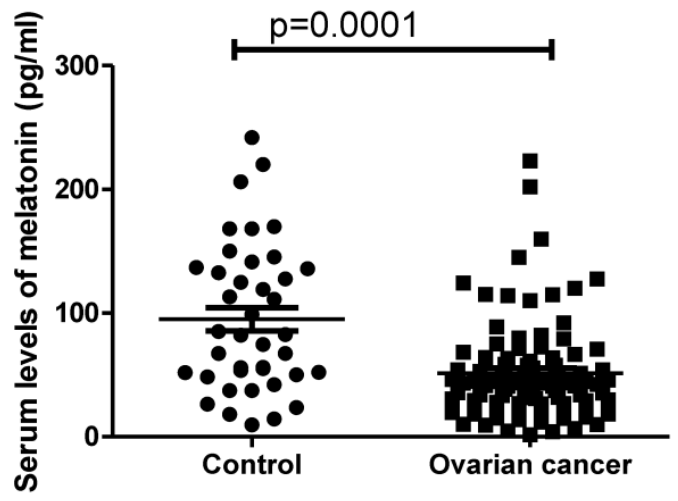

Figure 1: The serum levels of melatonin were significantly reduced in women with ovarian cancer at diagnosis compared to healthy women $(p=0.001)$.

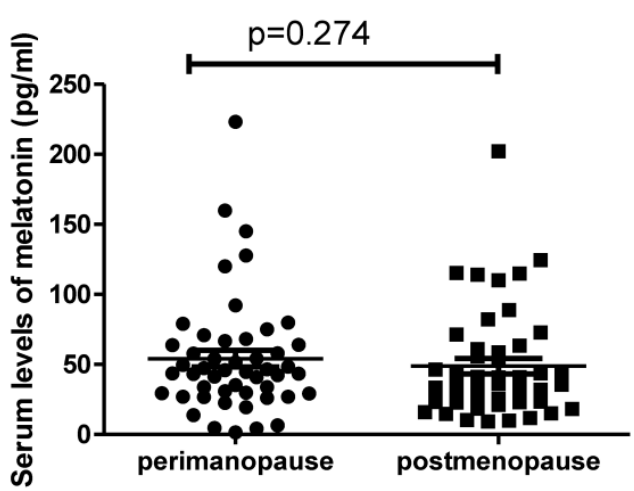

Figure 2: The serum levels of melatonin were not different between perimenopausal women with ovarian cancer and postmenopausal women with ovarian cancer $(p=0.274)$.

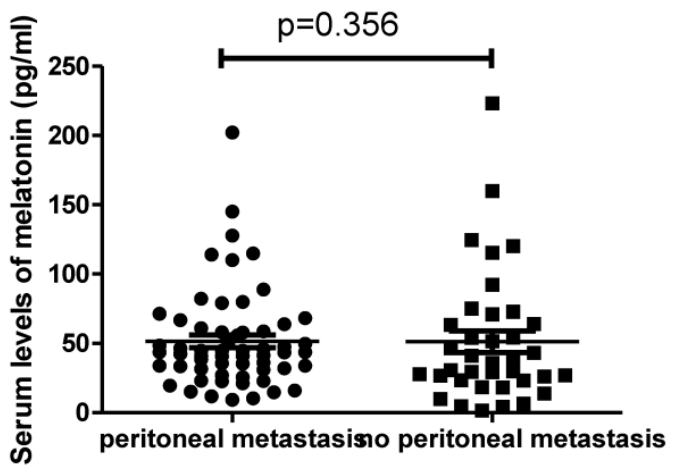

Figure 3: The serum levels of melatonin were not different between ovarian cancer patients with peritoneal metastasis and without peritoneal metastasis $(p=0.356)$. 


\section{The incidence of developing ovarian cancer is not associated with season of birth}

We then analysed the association between the incidence of developing ovarian cancer and season of birth. The total number of women with ovarian cancer was 277 between 2008 and 2014 and there was no statistical difference in seasonal distribution between ovarian cancer patients and the control group $(\mathrm{n}=1,076)$ (Table 2, $\mathrm{p}=0.136)$.

We then analysed whether the reduction in serum melatonin levels in women with ovarian cancer was associated with the season of birth (Table 3). Multiple liner regression analysis shows that although there was a significant difference in the circulating levels of melatonin between healthy women and women with ovarian cancer, the effect of season of birth in serum levels of melatonin in both healthy women and women with ovarian cancer was not significant $(p=0.60)$. Further analysis investigated the interaction effect of patient/control and season of birth. The result shows the interaction effect was not statistically significant $(p=0.71)$, suggesting there is no significant season of birth effect in the circulating levels of melatonin in both patients and controls, and the effect of ovarian cancer on melatonin level is the same across different seasons of birth.

Table 2: The analysis of season of birth in women with ovarian cancer between 2008 and 2014.

\begin{tabular}{lllll}
\hline & $\begin{array}{l}\text { Ovarian cancer } \\
\text { patients }(\mathrm{n}=277)\end{array}$ & $\begin{array}{l}\text { Controls } \\
(\mathrm{n}=1,076)\end{array}$ & Total & $\begin{array}{l}\text { Chi-square } \\
\text { test P value }\end{array}$ \\
\hline $\begin{array}{l}\text { Season } \\
\begin{array}{l}\text { Spring } \\
\text { (number, \%) }\end{array}\end{array}$ & $63(22.74 \%)$ & $237(22.03 \%)$ & $300(22.17 \%)$ & 0.136 \\
$\begin{array}{l}\text { Summer } \\
\text { (number, \%) }\end{array}$ & $53(19.13 \%)$ & $272(25.28 \%)$ & $325(24.02 \%)$ & \\
$\begin{array}{l}\text { Autumn } \\
\text { (number, \%) }\end{array}$ & $88(31.77 \%)$ & $332(30.86 \%)$ & $420(31.04 \%)$ & \\
$\begin{array}{l}\text { Winter } \\
\text { (number, \%) }\end{array}$ & $73(26.35 \%)$ & $235(21.84 \%)$ & $308(22.76 \%)$ & \\
\hline
\end{tabular}

Table 3: Circulating levels of melatonin $(\mathrm{pg} / \mathrm{ml})$ in women with ovarian cancer and controls by season of birth (median, range).

\begin{tabular}{lll}
\hline & \multicolumn{2}{l}{$\begin{array}{l}\text { Women with ovarian } \\
\text { cancer at diagnosis }(\mathrm{n}=96)\end{array}$} \\
\hline Spring & $43.3(9.9-223.1)$ & $78.4(52-150)$ \\
Summer & $40.8(15.1-120.1)$ & $75.4(93.7-241)$ \\
Autumn & $42.4(1.5-159.9)$ & $84.7(26.3-220)$ \\
Winter & $41.8(9.3-75.2)$ & $83.9(23.6-168)$ \\
\hline
\end{tabular}

\section{Discussion}

Ovarian cancer is the third most common gynaecological cancer and the leading cause of death in gynaecological cancers worldwide. Although the pathogenesis of ovarian cancer is not clear, a number of studies proposed that night shift work is associated with the increased risk of developing gynaecological cancers including breast cancer [5] and endometrial cancer [6]. One of the mechanisms of this association may be the lower melatonin production by pineal gland. Melatonin is secreted in a circadian pattern which is dependent on the length of darkness at night. Exposure to light at night reduced melatonin secretion. The reduction of serum levels of melatonin was seen in women with endometrial cancer [19].

Although night shift work does not appear to be associated with the increased risk of developing ovarian cancer, the association between melatonin and the risk of developing ovarian cancer has been suggested [20]. This prompted us to investigate whether the circulating levels of melatonin are associated with the pathogenesis of ovarian cancer. In our current study, our data shows that the circulating levels of melatonin were significantly lower in women with ovarian cancer compared to healthy women, regardless the classification of ovarian cancer. Melatonin is also produced by ovaries that regulate the function of the ovaries, in addition to the pineal gland $[9,10]$, and the reduction of melatonin levels impacts ovary morphology [16]. Supplementation of melatonin reduces incidence of ovarian cancer in ethanol-deprived rats [21]. Treatment with melatonin is also found to have additive effects on metastatic breast cancer in the combination with chemotherapy [22]. Therefore our data may further suggest that melatonin is involved in the development of ovarian cancer. Recently strong evidence suggested that melatonin contributes cancer prevention through its action of anti-oxidative stress and anti-angiogenic activities (reviewed in [13]). The anti-oxidative effects of melatonin have been suggested to be more pronounced in hormone-dependent cancers such as breast and ovarian cancer [11]. When the secretion of melatonin is suppressed, the cancer cells become more active. These studies suggested that melatonin is a modifier of cancer progression, in particular in hormone related cancers including ovarian cancer although our current data show that there is no significant difference in melatonin levels between patients with and patients without peritoneal metastasis.

In addition to anti-oxidative stress and anti-angiogenic activities by melatonin, melatonin also affects the secretion of estrogen. Ovarian cancer is a hormone related cancer and the increased estrogen level is involved in the development of ovarian cancer [23]. Exposure to light at night disrupts the natural melatonin-estrogen balance, resulting in less melatonin secretion than there should be, and more estrogen release. The net effect is a heightened risk of 
estrogen-sensitive tumors, such as breast cancer and ovarian cancer. A study has suggested that the reduction of circulating levels of melatonin is correlated with the development of endometrial cancer regardless of the status of menopause [19]. In our current study our data also show that there is no significant difference in the levels of melatonin in perimenopausal women and postmenopaual women. We also found that there is no correlation between the circulating levels of melatonin and estrogen in postmenopaual women with ovarian cancer.

There is growing evidence suggesting adult's life expectancy may be affected by season of birth [24, 25]. Season of birth may be an important environment factor to be associated with a number of diseases including gynaecological cancers [7] [26]. Thus, season of birth appears to be linked to morbidity and mortality in adults' life later. However, the mechanism of this association is unknown. Environmental factors such as circadian rhythm may be involved in this association. Therefore in this study we investigated whether the changes in melatonin level in circulation can explain the association between season of birth and cancers. The secretion of melatonin is suppressed in summer which has longer day length and this may result in an increased risk of developing gynaecological cancers [7]. In addition, increasing circulation levels of melatonin is associated with light exposure at birth [27]. In this current study we examined the association between the incidence of ovarian cancer and season of birth in the period of 7 years. With 277 women with ovarian cancer, however our data show that there is no association between the incidence of ovarian cancer and season of birth. We further found that the serum melatonin levels in women with ovarian cancer at diagnosis are not subject to the season of birth, although one study indicated that the secretion of melatonin is higher in winter and lower in summer than any other season in a healthy population [28]. This could be due to the difference in sample size.

There are some limitations in this study. The melatonin level was measured after ovarian cancer was diagnosed so that we do not know whether these women had lower melatonin before ovarian cancer was diagnosed. Whether the reduced level of melatonin is a causative or reflective response to ovarian cancer is unknown. However, treatment with melatonin reduced the incidence of ovarian adenocarcinomas in ethanol deprived rats [21] suggesting melatonin is likely to be involved in the development of ovarian cancer. In addition, because Wuxi Maternity and Children Health Hospital is a referral hospital, all the patients may not be born in Wuxi. Climate and daylight hours are variable between different regions. Ideally, the time for blood sample collection should be at night time without exposure to light conditions because previous studies suggested that morning melatonin levels (between 7 to $9 \mathrm{am}$ ) were five times higher than samples collected in the afternoon $[28,29]$.

In conclusion, we demonstrate a reduction of melatonin levels in women with ovarian cancer at diagnosis. There is no association between the incidence of ovarian cancer and season of birth, and melatonin level in women with ovarian cancer at diagnosis is not subject to season of birth. Future prospective studies are needed to confirm our findings in this study.

\section{Acknowledgments}

This study was supported by Chinese National Nature Sciences Foundation (Grant No. 81072130 to JX Ding). Authors would also like to thank all the patients who donated their blood for this study. Authors also would like to thank Victoria Rutten, from The University of Auckland for editing this manuscript.

\section{Disclosures}

None of the authors have a conflict of interest.

\section{References}

1. Edwards B.K., Brown M.L., Wingo P.A., Howe H.L., Ward E., Ries L.A.G., Schrag D., Jamison P.M., Jemal A., and Wu X.C. Annual report to the nation on the status of cancer, 1975-2002, featuring population-based trends in cancer treatment. Journal of the National Cancer Institute, 2005. 97(19): 1407.

2. Reiter R.J., Tamura H., Tan D.X., and Xu X.Y. Melatonin and the circadian system: contributions to successful female reproduction. Fertil Steril, 2014. 102(2): 321-8.

3. Lie J.A., Roessink J., and Kjaerheim K. Breast cancer and night work among Norwegian nurses. Cancer Causes Control, 2006. 17(1): 39-44.

4. Hansen J. Increased breast cancer risk among women who work predominantly at night. Epidemiology, 2001. 12(1): 74-7.

5. Davis S., Mirick D.K., and Stevens R.G. Night shift work, light at night, and risk of breast cancer. J Natl Cancer Inst, 2001. 93(20): 1557-62.

6. Viswanathan A.N., Hankinson S.E., and Schernhammer E.S. Night shift work and the risk of endometrial cancer. Cancer Res, 2007. 67(21): 10618-22.

7. Rowlands I.J., Weinstein P., Nagle C.M., Spurdle A.B., Webb P.M., Australian National Endometrial Cancer S., and Australian Ovarian Cancer S. Season of birth and risk of endometrial cancer. Asian Pac J Cancer Prev, 2011. 12(5): 1193-6.

8. Tan D.X., Manchester L.C., Liu X, Rosales-Corral S.A., Acuna-Castroviejo D. and Reiter R.J. Mitochondria and chloroplasts as the original sites of melatonin synthesis: a hypothesis related to melatonin's primary function and evolution in eukaryotes. J Pineal Res, 2013. 54(2): 127-38.

9. Glass J.D. and Knotts L.K. A brain site for the antigonadal action of melatonin in the white-footed mouse (Peromyscus leucopus): involvement of the immunoreactive GnRH neuronal system. Neuroendocrinology, 1987. 46(1): 48-55.

10. Itoh M.T., Ishizuka B., Kudo Y., Fusama S., Amemiya A., and Sumi Y. Detection of melatonin and serotonin N-acetyltransferase and hydroxyindole-O-methyltransferase activities in rat ovary. Mol Cell Endocrinol, 1997. 136(1): 7-13.

11. Hill S.M., Spriggs L.L., Simon M.A., Muraoka H., and Blask D.E. The growth inhibitory action of melatonin on human breast cancer cells is linked to the estrogen response system. Cancer Lett, 1992. 64(3): 249-56.

12. Cos S., Mediavilla M.D., Fernandez R., Gonzalez-Lamuno D., and Sanchez-Barcelo E.J. Does melatonin induce apoptosis in MCF-7 human breast cancer cells in vitro? J Pineal Res, 2002. 32(2): 90-6.

13. Viswanathan A.N. and Schernhammer E.S. Circulating melatonin and the risk of breast and endometrial cancer in women. Cancer Lett, 2009. 281(1): 1-7.

14. Blask D.E., Sauer L.A., and Dauchy R.T. Melatonin as a chronobiotic/anticancer agent: cellular, biochemical, and molecular 
mechanisms of action and their implications for circadian-based cancer therapy. Curr Top Med Chem, 2002. 2(2): 113-32.

15. Dauchy R.T., Xiang S., Mao L., Brimer S., Wren M.A., Yuan L., Anbalagan M., Hauch A., Frasch T., Rowan B.G., Blask D.E., and Hill S.M. Circadian and melatonin disruption by exposure to light at night drives intrinsic resistance to tamoxifen therapy in breast cancer. Cancer Res, 2014. 74(15): 4099-110.

16. Soares J.M., Jr., Simoes M.J., Oshima C.T, Mora O.A., De Lima G.R., and Baracat E.C. Pinealectomy changes rat ovarian interstitial cell morphology and decreases progesterone receptor expression. Gynecol Endocrinol, 2003. 17(2): 115-23.

17. Wurtman R.J., Axelrod J., and Chu E.W. Melatonin, a pineal substance: effect on the rat ovary. Science, 1963. 141(3577): 277-8.

18. Tamura H., Nakamura Y., Korkmaz A., Manchester L.C., Tan D.X., Sugino N., and Reiter R.J. Melatonin and the ovary: physiological and pathophysiological implications. Fertil Steril, 2009. 92(1): 328-43.

19. Grin W. and Grunberger W. A significant correlation between melatonin deficiency and endometrial cancer. Gynecol Obstet Invest, 1998. 45(1): 62-5.

20. Poole E.M., Schernhammer E.S., and Tworoger S.S. Rotating night shift work and risk of ovarian cancer. Cancer Epidemiol Biomarkers Prev, 2011. 20(5): 934-8.

21. Chuffa L.G., Fioruci-Fontanelli B.A., Mendes L.O,, Favaro W.J., Pinheiro P.F, Martinez M., and Martinez F.E. Characterization of chemically induced ovarian carcinomas in an ethanol-preferring rat model: influence of long-term melatonin treatment. PLoS One, 2013. 8(12): e81676.

22. Lissoni P., Barni S., Mandala M., Ardizzoia A., Paolorossi F., Vaghi M., Longarini R., Malugani F., and Tancini G. Decreased toxicity and increased efficacy of cancer chemotherapy using the pineal hormone melatonin in metastatic solid tumour patients with poor clinical status. Eur J Cancer, 1999. 35(12): 1688-92.

23. Lacey J.V., Jr., Mink P.J., Lubin J.H., Sherman M.E., Troisi R., Hartge P., Schatzkin A., and Schairer C. Menopausal hormone replacement therapy and risk of ovarian cancer. JAMA, 2002. 288(3): 334-41.

24. Reffelmann T., Ittermann T., Empen K., Dorr M., and Felix S.B. Is cardiovascular mortality related to the season of birth?: evidence from more than 6 million cardiovascular deaths between 1992 and 2007. J Am Coll Cardiol, 2011. 57(7): 887-8.

25. Doblhammer G. and Vaupel J.W. Lifespan depends on month of birth. Proc Natl Acad Sci U S A, 2001. 98(5): 2934-9.

26. Yuen J., Ekbom A., Trichopoulos D., Hsieh C.C., and Adami H.O. Season of birth and breast cancer risk in Sweden. Br J Cancer, 1994. 70(3): 564-8.

27. Jaldo-Alba F., Munoz-Hoyos A., Molina-Carballo A., Molina-Font J.A., and Acuna-Castroviejo D. Light deprivation increases plasma levels of melatonin during the first $72 \mathrm{~h}$ of life in human infants. Acta Endocrinol (Copenh), 1993. 129(5): 442-5.

28. Nogueira L.M., Sampson J.N., Chu L.W Yu K, Andriole G., Church T, Stanczyk F.Z., Koshiol J., and Hsing A.W. Individual variations in serum melatonin levels through time: implications for epidemiologic studies. PLoS One, 2013. 8(12): e83208

29. Hsing A.W., Meyer T.E., Niwa S., Quraishi S.M., and Chu L.W. Measuring serum melatonin in epidemiologic studies. Cancer Epidemiol Biomarkers Prev, 2010. 19(4): 932-7. 